\title{
Apagón eléctrico como puerta al aprendizaje basado en proyectos en una escuela primaria argentina: el papel fundamental de los docentes
}

\section{Power outage and project-based learning in an Argentine primary school: the key role of teachers}

\author{
Fanaro, María de los Ángeles; Suasnábar, Juan; Elgue, Mariana
}

María de los Ángeles Fanaro Sobre la autora mariangelesfanaro@gmail.com Universidad Nacional del Centro de la Provincia de Buenos Aires (NEES - UNCPBA), Argentina Juan Suasnábar Sobre el autor juansuas@gmail.com Universidad Nacional del Centro de la Provincia de Buenos Aires (NEES - UNCPBA), Argentina

Mariana Elgue Sobre la autora nanaelgue@gmail.com Universidad Nacional del Centro de la Provincia de Buenos Aires (UNCPBA), Argentina

Itinerarios educativos

Universidad Nacional del Litoral, Argentina

ISSN: $1850-3853$

ISSN-e: 2362-5554

Periodicidad: Anual

vol. 1, núm. 14, 2021

revistadelindi@fhuc.unl.edu.ar

Recepción: 29 Octubre 2020

Aprobación: 26 Julio 2021

URL: https://doi.org/10.14409/ie.2021.14.e0006
Resumen: En este trabajo se analiza una experiencia de Aprendizaje Basado en Proyectos (ABP) en una escuela primaria argentina generado a partir de un apagón eléctrico masivo y extendido en el tiempo. Se realizó una inmersión en el campo con observaciones no participantes en reuniones del equipo docente, en clases dentro y fuera del aula. Se presenta una narración del proyecto desde su gestación hasta el cierre, identificando en cada momento las señas de identidad propias del ABP reflexionando sobre su puesta en juego en la dinámica escolar. Se concluye sobre el papel destacado de las actitudes y las acciones de los docentes, como condicionantes para el desarrollo de un ABP, siendo ellos los actores clave de la innovación en el aula.

Palabras clave: ABP, innovación pedagógica, docente, escuela primaria.

Abstract: This paper deals with a Project Based Learning (PBL) experience in an Argentine primary school that was the result of a massive and extended power outage. Our immersion in the field involved non-participant observations in teachers' meetings and in classes either inside or outside the classroom. We provide a description of the whole project development, identifying all hallmarks of PBL and reflecting on how it operates in the school dynamics. A conclusion is drawn on the outstanding role of teachers' attitudes and actions as conditioning factors for PBL development, and thus, on teachers as key actors in class innovation.

Keywords: PBL, pedagogical innovation, teacher, primary school.

\section{Introducción y estado de la cuestión}

Hernández (2002) y Vergara (2016) proponen entender al enfoque por proyectos como algo que va más allá de una metodología, una pedagogía o una fórmula didáctica basada en una serie de pasos. Así, se invita a reconocer que el aprendizaje es una práctica emocional, además de cognitiva y comportamental, 
lo cual requiere una nueva mirada a la escuela en su totalidad, considerando primordialmente que la educación en general es un problema político (Terigi 2006, 2008; Carbonell Sebarroja, 2015; Masferrer y Baqueró, 2014). Por su parte, el ABP además de conllevar incidencia en la visión política, educativa, curricular, implica profundos cambios en la mirada del mismo conocimiento: se requiere construir una nueva noción de vínculo con los alumnos, enfatizando en otras posibilidades de experiencias de enseñanza y aprendizaje y naturalizando el proceso de enseñanza como un espacio de incerteza, donde no todo está previsto, y que esto, lejos de ser un obstáculo, sea el motor de la búsqueda de conocimientos (Larrosa, 2006)

Carbonell Sebarroja (2015) y Vergara (2016) esclarecen el panorama dando una idea de lo que no implica el ABP: no necesariamente implica una actividad interdisciplinar, ni el uso de tecnologías, ni es un modelo único de enseñanza (en el sentido de tener que trabajar todo el tiempo por proyectos) ni es algo que los docentes diseñen y los estudiantes realizan. Lo esencial es trabajar desde, con y para la realidad: no se hacen supuestos sobre una hipotética realidad -lejana y totalmente desconocida para los estudiantes- de la que se supone un problema a resolver: se trabaja directamente con una situación concreta, con cifras reales $y$, a ser posible, en la que habitan los alumnos (Carbonell Sebarroja, 2015). La perspectiva epistemológica acerca de la construcción del conocimiento por parte de los estudiantes requiere deconstruir la idea de generar situaciones artificiales para conseguir que los alumnos aprendan. Lo esencial es que los conocimientos disciplinares, resulten útiles para resolver inquietudes de los estudiantes: que permita responder a sus dudas en torno a un tema o amplíe el marco de análisis sobre un eje temático que se plantee.

Se puede notar que en la literatura acerca del ABP -apenas esbozada en este trabajo por cuestiones de espacio- se enfatiza el protagonismo y la actividad de los estudiantes en los proyectos. Monel y Carbajo (2011) y Caballero, Briones y Flores (2014) en sus trabajos se enfocan en los docentes y el ABP. En esta investigación, sin embargo, se busca comprender cómo se posicionan los docentes respecto al saber y al estudiante para co-construir un proyecto con sus estudiantes y llevarlo a cabo, y cuáles son las acciones concretas que deben realizar, que sinérgicamente encastren con las acciones de sus colegas y estudiantes para que el proceso de enseñar y aprender por proyectos pueda llevarse a cabo.

\section{Materiales y métodos}

Se realizó una inmersión en el campo durante un tiempo de ocho semanas, cubriendo desde la gestación del proyecto, hasta la presentación del producto final por parte de los estudiantes. Se realizaron las observaciones no participantes en cinco reuniones de dos horas de duración semanal con el equipo de docentes, en diez momentos del desarrollo del proyecto (esto incluye las clases del docente de año y de clases de áreas de música y ciencias de la computación y en dos salidas educativas fuera de la escuela) registrando en forma de notas y en audio. La selección del Colegio y del curso fue intencional, ya que se trata de un colegio público de gestión privada de una formación reciente de la ciudad de Tandil, Buenos Aires (Argentina) que se puede considerar que realiza innovación educativa ya que los cambios que se generan no son sólo algunas actividades 
aisladas y ocasionales, sino que forman parte de la cotidianeidad de las prácticas y el contexto escolar, donde explícitamente las bases pedagógicas del Colegio se dirigen al ABP. También es característico de esta institución la formación de redes de intercambio y cooperación entre los docentes, junto a un asesor pedagógico interno de presencia continua en el colegio, una directora, y apoyos externos, que son solicitados cuando la situación lo demanda. La escuela se estructura por años escolares, con un docente de aula (con quienes están la mayor parte del tiempo) y profesores de materias especiales (música, inglés y ciencias de la computación).

El armado y la planificación de los proyectos en esta institución se realiza en forma colaborativa por todo el personal de la institución (maestras de grado, profesores de áreas especiales) en reuniones semanales de dos horas de duración aproximadamente de equipo en las que participan también los directivos y el asesor pedagógico. En estas reuniones, se tratan cuestiones de índole institucional en general, y luego los docentes por curso dedican un tiempo al tratado del ABP. Por su parte se seleccionó el cuarto grado porque este era el primer curso del Colegio que desde el principio trabaja por proyectos, es decir para estos estudiantes lo natural en la escuela era trabajar por proyectos.

\section{El proceso vivenciado desde la gestación del proyecto hasta su cierre}

Se presenta una narración organizada en lo que se consideran elementos clave del desarrollo del ABP:

El apagón masivo en Argentina: construyendo el interés común a través de la conexión con el mundo social de estudiantes y docentes

Una de las actividades que conforman las características del colegio es la realización de una conversación cotidiana entre cada docente y su grupo de estudiantes, donde se tiene la oportunidad de contar experiencias, ideas o situaciones vividas, que consideren interesante comunicar a sus pares. Este tiempo, que se da como inicio de la jornada escolar (duración aproximada de 15 minutos), resulta esencial para percibir los intereses y necesidades de los estudiantes. Para poder plantear un proyecto, debe entenderse que se trata de establecer un interés «común», compartido. Es decir, se trata de establecer entre ambas partes, un interés que no es personal y privado sino que sea un asunto de todos, por ejemplo del barrio, la comunidad, la ciudad. En este sentido, Larrosa (2018) alerta de que «no es posible que la escuela parta del interés de los estudiantes, a no ser que haya un desplazamiento que convierta ese interés en un asunto común» (100). Se trata de construir un interés de todos, que concierne a todos. El autor sostiene que se trata de construir un asunto «colocado entre todos», haciendo referencia al origen etimológico de la palabra interés, ya que lo que tiene interés es lo que es o está entre, en medio, lo que ya es de todos en general y, por tanto, de nadie en particular. En palabras de Masschelein y Simons (2013) convertir algo en objeto de interés común significa volverlo público, disponible para todos. El docente tiene un rol fundamental en este momento, pues es el encargado de 
tomar las palabras de sus estudiantes, y convertirlas en un «asunto para hablar», para pensar, donde todos dirijan la mirada y la atención, es decir para enseñar. En este sentido, es preciso ser cuidadosos con la idea de que una pedagogía activa o innovadora parte de los intereses de los estudiantes. El interés no es un elemento dado a priori, ni potestad de uno o varios sujetos; es la resultante -siempre provisoria- de una construcción colectiva y dialógica. Por ello mismo, tampoco es una cuestión aritmética, en el sentido de «el interés de la mayoría». El interés común supone una larga conversación, propuestas, idas y vueltas, durante las cuales el rol de coordinador del docente es fundamental.

En el caso del proyecto en análisis, una de las cuestiones que tuvo más resonancia dentro de las conversaciones en diferentes rondas fue la hipotética posibilidad de que el apagón se prolongara en el tiempo, lo que motivó a pensar sobre la necesidad de considerar formas alternativas de obtener energía para superar las dificultades vividas. Los docentes manifestaron la importancia de detectar la recurrencia de ciertos temas en los momentos de ronda con los estudiantes al inicio de cada día, tanto como el efecto que tienen en el grupo (a veces un alumno o alumna comenta algo que para el resto de sus pares no resultaba una cuestión presente, y ello despierta una inquietud en otros), de la escucha de sus inquietudes en los demás momentos de la clase, y del diálogo entre los docentes para compartir sus percepciones acerca de las temáticas que posibilitarían la realización de un proyecto. Aquí se destaca que no se trata de cualquier escucha, sino en el mismo sentido que el concepto que sostienen Sierra y Blanco (2017) de la escucha como investigación de la experiencia educativa. Esta escucha requiere un ejercicio difícil, que supone un desplazamiento de la atención: del docente y lo que está buscando y de lo que piensa (y siente) sobre eso, a lo que puede que no conozca, a lo que es nuevo, a lo que no había pensado previamente, a lo que puede descolocarlo o conmoverlo. Así, esto no es algo que simplemente sucede, sino que requiere de un ejercicio consciente y sostenido para reconocer que la escucha es una oportunidad de transformación, de oportunidad para aprender.

\section{Ingresando el interés común como objeto de enseñanza: interacciones entre los docentes para planificar tiempos, contenidos, lugares}

En la primera reunión de docentes, ellos consensuaron que el tema del apagón masivo reciente había sido el que había estado presente reiteradamente en las rondas, comentando algunas expresiones de los estudiantes, y así comenzaron a conversar y acordar de qué forma se ingresará el interés común establecido, como objeto de enseñanza. En ese momento los docentes revisaron los contenidos curriculares que podrían trabajarse con esta idea de proyecto. Así, en una especie de «tormenta de ideas» los docentes de distintas áreas proponen trabajar los distintos tipos de energía, enfatizando en la clasificación entre renovables y no renovables, la realización de una maqueta de la ciudad, la construcción de una habitación autosustentable, la generación de energía eléctrica en forma alternativa, estudio de los conceptos de electricidad, magnetismo, etc. Las conversaciones de los docentes giraban en torno al propósito del proyecto, a los contenidos curriculares a abordar y a las posibles actividades para los estudiantes. 
Para la segunda reunión, el debate continuaba con el enriquecimiento de los docentes de áreas especiales, y al finalizar este encuentro los docentes acordaron que el proyecto se focalizaría en la generación y el uso de la energía eléctrica; en ese momento también declararon la necesidad de disipar sus dudas conceptuales con asesores especialistas en la temática, quienes clarificaron conceptos en un espacio y tiempo dentro de la reunión semanal siguiente (tercera reunión). Volver la problemática planteada como objeto de enseñanza requiere por parte de los docentes, una mirada flexible ante el curriculum, y esfuerzo por lograr una armonía entre los conceptos e ideas del proyecto y los contenidos curriculares. El diálogo entre ellos fue un aspecto clave, tanto en las reuniones semanales, como en los recreos, e incluso utilizando fuentes no formales, conversaciones privadas, como redes sociales. Esos espacios de intercambio y construcción colectiva aparecen como un pilar para la construcción de proyectos a su vez que como uno de los desafíos más importantes. En particular los profesores de las materias especiales fueron quienes reclamaron la poca permanencia de tiempo en la escuela como tiempo para compartir con los demás docentes, pero a su vez reconocieron que es una imposibilidad poco probable de mejorar en las condiciones de la docencia en Argentina.

\section{Recalculando constantemente el rumbo: evaluación permanente del avance del proyecto}

En la tercera reunión el equipo docente planteó la importancia de tratar con los estudiantes las relaciones entre energía y sociedad, la energía en la vida cotidiana y plantearon algunas actividades que se podían realizar en esa línea. En esa semana el docente de aula planteó el proyecto a los estudiantes, con la posibilidad de generar como producto final (el cual había sido acordado con el resto del equipo docente) distintos prototipos de artefactos que permitan generar energía eléctrica de manera independiente de la red eléctrica doméstica. Los estudiantes, entusiasmados con la propuesta, acordaron con el docente llamar a este proyecto «La fábrica de inventos».

El docente de aula propuso reconocer las implicancias sociales del apagón, más allá de las repercusiones personales. Para ello, se propusieron lecturas de diferentes artículos periodísticos donde se hizo referencia a la causa (fallo en el sistema de interconexión eléctrica) y sus consecuencias (en el tránsito y el transporte, la seguridad, el comercio, en pacientes electrodependientes, etc.). En aula analizaron el recorrido de la energía eléctrica desde donde se genera (centrales eólicas, nucleares, hidroeléctricas, solares) hasta llegar a los usuarios finales, en primer lugar, mediante un mapa que muestra la red SADI (Sistema Argentino de Interconexión Eléctrica), para luego entrar en más detalle y estudiar las diferentes instancias de reducción de voltaje. Como parte de la consigna comprendía utilizar recursos naturales renovables para la generación de energía, el docente les propuso a los estudiantes indagar sobre algunos inventos antiguos que conocieran, para comprender los recursos empleados para su funcionamiento, teniendo en cuenta sus mecanismos. Utilizando referencias históricas, se fueron adentrando en los principios fundamentales de los generadores de energía eléctrica, para luego abordar los conceptos de electricidad estática, corriente eléctrica y magnetismo, mediante recreaciones 
de los experimentos realizados por las distintas personalidades de la ciencia, y también con recursos virtuales como las simulaciones, que ofrecieron la posibilidad de interpretar aspectos no visibles de estos fenómenos (como la interacción entre cargas eléctricas o las líneas de fuerza en los campos magnéticos), ayudados también con representaciones gráficas.

En las últimas semanas del proyecto, el docente de aula retomó algunos aspectos de la representación y su relación con la realidad, dando así la oportunidad de proponer a sus estudiantes nociones relativas a la construcción y propiedades de la circunferencia y del triángulo, siempre en el contexto del problema. También en el momento de formular el texto que acompañaría a las presentaciones de los prototipos inventados, el docente trabajó contenidos relativos al área del diseño curricular de Prácticas del lenguaje.

En cuanto al área de Ciencias de la Computación, la profesora propuso trabajar sobre los inventos y la tecnología, diferenciando elementos naturales de artificiales, orientados a componentes electrónicos y eléctricos. Para abordar algunas nociones básicas de energía eléctrica, la profesora permitió experimentar con circuitos con corriente continua y algunos componentes electrónicos básicos, ofreció un voltímetro para realizar mediciones de diferencia de potencial de baterías. Esto permitió a los estudiantes hipotetizar resultados, realizar cálculos aproximados con la unidad de medida de la diferencia de potencial, y comprobarlos experimentalmente. En diálogo con el docente de clases, y en conocimiento de la presentación de la producción final que harían los estudiantes, la profesora ofreció el uso de software para la creación de presentaciones, que les permitió elaborar las diapositivas utilizadas en la presentación final del proyecto ante el auditorio.

En el área de Música, el profesor propuso conocer un poco más sobre qué es lo que sucede con la energía sonora. Si bien es algo que no se utiliza para generar otros tipos de energía, es una de las más importantes, ya que el sonido es la principal herramienta de comunicación humana. Indagaron entonces sobre cómo funcionan los instrumentos musicales, empezando por la propia voz, de manera de poder entender cómo se produce el sonido. A partir de estas ideas los estudiantes construyeron nuevos instrumentos musicales con materiales reciclados.

Es notorio el esfuerzo tanto del docente de aula como de los profesores especiales, por hacer que los estudiantes conecten lo que aprenden con su vida, con su realidad. Para Vergara (2016) este es uno de los pilares del aprendizaje por proyectos, y nuevamente cobra importancia el papel vital de los docentes en interacción: fueron ellos quienes plantearon esas experiencias a sus colegas en las reuniones, los que permanentemente están cuestionando y repreguntándose acerca de los avances del proyecto, de las acciones y decisiones a tomar. Así, la planificación y desarrollo de las propuestas de clase, no es sólo una hipótesis de trabajo, en el sentido de que es una proyección que se pone a prueba en cada encuentro con los estudiantes, sino que es una y múltiple, una construcción que es de un colectivo de docentes que piensa, debate, intercambia, vivencia, alterna posiciones (a veces como profesor, a veces como 'estudiante' de las propuestas de sus colegas), reflexiona y propone cursos de acción. 


\section{Aprendiendo fuera del aula}

Cuando el proyecto ya había comenzado a andar, los docentes planificaron dos salidas: a la usina de energía eléctrica de la ciudad y a una casa totalmente autosustentable de la ciudad, denominada «La osera». En ambas visitas los docentes acompañaron a sus estudiantes, y mantuvieron la actitud de curiosidad y apertura, puesto que se trata de dos lugares de visita poco común. En la visita a la Usina, los estudiantes cuestionaron acerca de la generación de la electricidad en nuestro país, su distribución, el trabajo de los operarios de la Usina, los costos de producción y distribución, la seguridad en el uso de la electricidad en sus hogares, los cableados subterráneos, las unidades de la corriente eléctrica, etc. Luego se realizó la visita a «La Osera de la Sierra» que constituye un espacio sustentable, diseñado y construido con materiales naturales, donde los estudiantes también realizaron numerosas e interesantes preguntas.

El aprender fuera del aula es un aspecto destacado en el ABP, porque permite trascender el enseñar como una tarea individual, sectorizada. Como sostienen (Birgin y Terigi, 1998) es común que al conocimiento se lo apropie primero un docente para luego impartirlo a sus alumnos de manera individual, olvidando que también puede ser enseñado por un colectivo de docentes y por otros sectores de la sociedad que desde su lugar y a su manera, aportan a la enseñanza. En el marco de la propuesta del Colegio, buscar lugares donde ir, personas que invitar o visitas a realizar no es una opción, está planteado como imperativo. Lo que se busca es hacer un ida y vuelta entre el adentro y el afuera de lo escolar. Hay un momento que es de ingreso de algo que sucede en 'la realidad' (eso que llamamos la construcción del interés común, el tornar público un asunto) en que algo que sucede fuera de la escuela ingresa, y es tomado y construido como objeto de enseñanza y de aprendizaje. Pero hay un momento de retorno, de volver a conectar eso que se planificó con el afuera, con el entorno cercano. Se busca, como ya decía Freinet $(1969,41)$ de «no separar la escuela de la vida».

\section{Construcción colectiva del cierre y preguntas para futuros proyectos}

La decisión de la producción final no es algo que los profesores deciden antes de proponer el proyecto a los estudiantes, esto también está sujeto a cambios de rumbo, ya que los profesores consideran que el proceso de producción es muy importante para el aprendizaje, además de ser lo que inspira y guía la realización del proyecto. No obstante, definir el producto y el punto de corte es una decisión central ya que establece los límites del proyecto, qué preguntas (las que siempre son infinitas) y qué conocimientos serán incluidos y cuáles quedarán afuera. Una decisión que va aparejada con el producto es la decisión de su exposición: una comunicación útil, que permita enseñar a los demás todo lo trabajado.

En este caso, los estudiantes decidieron mostrar a sus padres lo trabajado. El desafío de diseñar un prototipo de artefacto que satisfaga una necesidad utilizando energías renovables implicó para los estudiantes y los docentes poner en juego todos los saberes trabajados a lo largo del proyecto. Lo esencial era abordar los conceptos, sin la presión de si luego esos artefactos al construirlos funcionarían realmente. Desde el área de música también mostrarían los 
instrumentos construidos, explicando sus elementos. Los estudiantes realizaron una presentación oral de sus esquemas de prototipos, ayudados por diapositivas diseñadas por ellos mismos, indicando las partes componentes de sus prototipos inventados, y el funcionamiento de los mismos, ante sus pares, padres y directivos del colegio, y personas calificadas (las mismas que habían disipado las dudas de los docentes al comenzar el proyecto) quienes realizaron las devoluciones pertinentes.

\section{Reflexiones finales}

Este trabajo plantea la necesidad del estudio de la formación docente, como uno de los pilares del funcionamiento del ABP. En primer lugar, la bibliografía acerca del ABP en la escuela resalta que el protagonismo de los estudiantes es clave, entendido como el motor y el propósito de la enseñanza. A partir de la inmersión en el campo es posible concluir que este protagonismo tuvo lugar en la mayoría de los momentos, puesto que ellos plantearon las preguntas, las temáticas, la escucha. Sin embargo, este protagonismo corre paralelo al de los docentes, quienes, sostuvieron actitudes de apertura al diálogo y a la mirada de sus pares, de sus estudiantes y de otros actores de la enseñanza, que no siempre se encuentran dentro de la escuela. En este sentido, se hace imprescindible que los docentes formen un equipo de trabajo, y que cuenten tanto con el espacio como con el tiempo para plantear ideas, dudas, y recibir el apoyo de la institución para que ese proyecto gestado pueda llevarse adelante.

Si bien el objetivo de este trabajo no fue realizar un análisis crítico sobre el impacto -conflictos, obstáculos, contradicciones- de la propuesta, considerada innovadora en un sistema de enseñanza tradicional, podemos destacar la incidencia en el aprendizaje de las actitudes, tanto de los estudiantes como de los docentes. Entre ellos, destacamos el aprender a escuchar, a esperar y a tener autoconfianza dentro y fuera del aula, que son muy valorables para sortear los obstáculos que representa esta forma particular de acercarse al saber.

En síntesis, una de las piezas fundamentales de este proceso y que no siempre es considerada como tal, es la comunicación entre los docentes para que la metodología sea activa, activa para estudiantes y docentes. Los docentes como equipo y apoyados en (y en conjunto con) la institución donde trabajan, son quienes tienen entre sus manos la posibilidad de realizar la innovación en su cotidiano, la que no es ocasional, ni para unos pocos estudiantes. El gran desafío es encontrar la forma de fomentar esta comunicación y acentuar el mecanismo de trabajo docente en equipo.

\section{Referencias}

Birgin, A. y Terigi, F. (1998). Proyectos escolares y formación docente: Una nueva oportunidad para pensar viejos problemas. Pensamiento Educativo. 23, 165-188.

Caballero, E.; Briones, C. y Flores, J. (2014). El aprendizaje basado en proyectos y la autoeficacia de los/las profesores/as en la formulación de un plan de clase. Alteridad Revista de Educación.https://doi.org/10.17163/alt.v9n1.2014.05

Carbonell J. (2014). La aventura de innovar, El cambio en la escuela. Madrid: Editorial Morata. 
Carbonell J. (2015). Pedagogías del siglo XXI, alternativas para la innovación educativa. Barcelona: Octaedro.

Freinet, C. (1969). Técnicas Freinet de la escuela moderna. México D.F.: Siglo XXI Editores.

Hernández, F. (2002). Los proyectos de trabajo. Mapa para navegantes en mares de incertidumbre. Cuadernos de Pedagogía 310.

Hernández, F., y Ventura, M. (1992). La organización del currículum por proyectos de trabajo. Barcelona: Graó.

Larrosa, J. (2006). Aloma: revista de psicologia, ciències de l'educació i de l'esport Blanquerna, 19.

Larrosa. J. (2018). P de profesor. Ciudad Autónoma de Buenos Aires. Centro de publicaciones Educativas y material didáctico.

Masschelein, J. y Simons, M. (2013). Em defesa da escola. Uma questao pública. Belo Horizonte: Autêntica Editora.

Masferrer, F.y Baqueró, M. (2014). 8 ideas Clave. Los proyectos interdisciplinarios. Barcelona: Graó.

Monel, F y Carbajo, M. (2011). Trabajo por proyectos, ¿y los docentes? Cuadernos de Pedagogía, 413.

Sierra Nieto, J. E., y Blanco García, N. (2017). El aprendizaje de la escucha en la investigación educativa. Qualitative Research in Education, 6(3), 303-326. http:/ /doi.org/10.17583/qre.2017.2783

Terigi, F. (2006). La enseñanza como práctica política. Diálogos Pedagógicos, IV (7).

Terigi, F. (2008). Los cambios en el formato de la escuela secundaria argentina: por qué son necesarios, por qué son tan difíciles. Propuesta Educativa. 29 (15) https://bi t.ly/2JgAgMN

Vergara, J. (2016). Aprendo porque quiero. El aprendizaje basado en proyectos paso a paso. Madrid: SM

\section{Notas}

[1] Doctora en Enseñanza de las Ciencias por la Universidad de Burgos (ESPAÑA). Profesor Adjunto en la Facultad de Ciencias Exactas de la UNCPBA, e Investigador Independiente del CONICET, con lugar de trabajo en el Núcleo de Estudios Educacionales y Sociales (NEES) de la Facultad de Ciencias Humanas Universidad Nacional del Centro de la Provincia de Buenos Aires (UNCPBA). Líneas de Investigación: Didáctica de las Ciencias Naturales, Didáctica de la Matemática. Formación Docente. mariangelesfanaro@gmail.com

[2] Especialista en Ciencias Sociales con mención en Psicoanálisis y prácticas SocioEducativas, FLACSO (ARGENTINA). Núcleo de Estudios Educacionales y Sociales (NEES) de la Facultad de Ciencias Humanas Universidad Nacional del Centro de la Provincia de Buenos Aires (UNCPBA). Líneas de investigación: Políticas Educativas, Derecho a la Educación, Formación Docente. juansuas@gmail.com

[3] Doctora en Enseñanza de las Ciencias (mención Física) por la Universidad Nacional del Centro de la Provincia de Buenos Aires (UNCPBA). Líneas de Investigación. Didáctica de las Ciencias Naturales, Formación Docente. nanaelgue@gmail.com 


\section{Notas de autor}

Sobre la autora

Doctora en Enseñanza de las Ciencias por la Universidad de Burgos (ESPAÑA). Profesor Adjunto en la Facultad de Ciencias Exactas de la UNCPBA, e Investigador Independiente del CONICET, con lugar de trabajo en el Núcleo de Estudios Educacionales y Sociales (NEES) de la Facultad de Ciencias Humanas Universidad Nacional del Centro de la Provincia de Buenos Aires (UNCPBA). Líneas de Investigación: Didáctica de las Ciencias Naturales, Didáctica de la Matemática. Formación Docente.

Sobre el autor

Especialista en Ciencias Sociales con mención en Psicoanálisis y prácticas Socio-Educativas, FLACSO (ARGENTINA). Núcleo de Estudios Educacionales y Sociales (NEES) de la Facultad de Ciencias Humanas Universidad Nacional del Centro de la Provincia de Buenos Aires (UNCPBA). Líneas de investigación: Políticas Educativas, Derecho a la Educación, Formación Docente.

Sobre la autora

Doctora en Enseñanza de las Ciencias (mención Física) por la Universidad Nacional del Centro de la Provincia de Buenos Aires (UNCPBA). Líneas de Investigación. Didáctica de las Ciencias Naturales, Formación Docente. 